\title{
Contrast Agent Started Identifier
}

National Cancer Institute

\section{Source}

National Cancer Institute. Contrast Agent Started Identifier. NCI Thesaurus. Code C69276.

An identifier for a contrast agent for which administration has started. 